\title{
Augmented reality to aid retention in an African university of technology engineering program
}

\author{
Nicolaas Luwes ${ }^{1}$, Leanri Van Heerden ${ }^{2}$ \\ ${ }^{1}$ Department of Electrical and Computer Engineering, Central University of Technology, \\ Free State (CUT), South Africa, ${ }^{2}$ Centre for Innovation in Learning and Teaching, Central \\ University of Technology, Free State (CUT), South Africa.
}

\begin{abstract}
Whenever complex threshold concepts are covered facilitators need to take extra care that students retain the new knowledge for future application. The problem encountered was that certain concepts, like digital logic gates with its layers of information are harder to retain. The purpose of this paper is to investigate the students' perceptions on the possible use of a custom design Quick Response code and Augmented Reality application in an Electrical Engineering subject. Evaluation was done on Logic Design III a 2nd year subject where the threshold concepts should have been imprinted. An exploratory design is implemented with data collected using an online survey tool. The results were promising as $88.89 \%$ of students felt that they will remember the content better with the use of the application and $83.33 \%$ were positive about adopting the technology in class. The paper shows that technology like Quick Response code and Augmented Reality in the classroom is not only beneficial for improving retention but is also welcomed by students.
\end{abstract}

Keywords: augmented reality; educational technology; logic gates; electrical engineering. 


\section{Introduction}

Edgar Allen Poe said that "To observe attentively is to remember distinctly" (Cave, 2019). A university facilitator's main aim is for their students to remember distinctly and are therefore always researching ways of helping students retain their skills and knowledge learned in class. The challenge comes within where students have to observe attentively.

In the subject Logic Design III at the Central University of Technology it was found that students have trouble remembering the threshold concept of logic gates. The concept of logic gates was first introduced in the first semester with basic electronic fundamentals and then revisited in semester 4 with Logic Design III. It was clear that the concept had to be revised in depth for students, to be able to continue with advance theories; and this raised concern over why students were not retaining this valuable information and how to address it.

The aim of this paper is to discuss the perspectives of students of a custom designed and programmed Quick Response code (QR) and an Augmented Reality (AR) application that was introduced in a lesson on logic gates. The AR was experienced through a smart phone application that showed students the operation, different manufacturing layers and types of logic gates - to assist in circuit design and understanding. The paper looks specifically at students' opinions of their retention of information using the QR and AR program.

Firstly, a literature review will look at retention and its relation to visual stimulation and AR. The context and AR activity are then elaborated on after which the methods and results are discussed. Finally, the paper is concluded with a discussion on the possible implications of the research.

\section{Literature review}

Preparing a student for the world of work means not only to provide them with the necessary skills and knowledge but also the ability to retain it for future application. The onus of retention does not only lie with the student but the lecturer as well. As Halpern and Hakel (2003) states: "What learners do determines what and how much is learned, how well it will be remembered, and the conditions under which it will be recalled" and what students do during the learning experience is up to the lecturer. Therefore, lecturers spend most of their time out of class researching different ways in which students acquire and retain new skills.

Seeing is believing isn't just an exclamation we use daily. Many studies have been conducted to show the correlation between visual feedback and the acquisition and retention of new skills and knowledge; in particular motor tasks (Batcho, Gagne, Bouyer, Roy, \& Mercier, 2016). The idea is that if the outcome predicts a student will be able to dig a hole, instead of having an in-depth theoretical discussion on soil and shovels, just have students dig a hole and see the results for themselves. Then when the theory is discussed the student has visual 
and other sensory experiences to connect it to. There are many ways to have students perform tasks in class and technology makes it easier all the time.

AR a key stone in Industry 4.0 (Alloghani, et al., 2018), with its potential for layering information in a 3-dimensional space, it is a perfect tool for creating an in-class visual learning experience. AR is introduced as a tool to train industrial operators (Nazir, Totaro, Brambilla, Colombo, \& Manca, 2012). AR expands on the existing space by overlaying objects and information that can be viewed through a smart device, resulting in a mixed reality (Chen, Liu, Cheng, \& Huang, 2016). Using AR tools in class will not only enhance the learning experience, but also the retention of the skills for future application.

\section{Context}

Central University of Technology (CUT) is a small university of technology catering mainly for students in the central region of South Africa. The department of Electrical, Electronic and Computer Engineering trains students for industry especially designing of electronic circuitry. One of the key threshold concepts in the discipline is logic gates, but it was found that when students revisited the concept in semester 4 , their retention from semester 1 was very poor. As the concept of logic gates is complex with many layers an intervention, it was necessary to help students remember for later application.

\section{Augmented reality activity}

The main objective is to use a new method to visually introduce the theory of logic gates and their manufacturing layering process. There are seven logic gates namely (Floyd, 2015); NOT, AND, NAND, OR, NOR, XOR and XNOR. Each one has a function, symbol, truth table and Boolean equation. See the example of the AND gate below: 


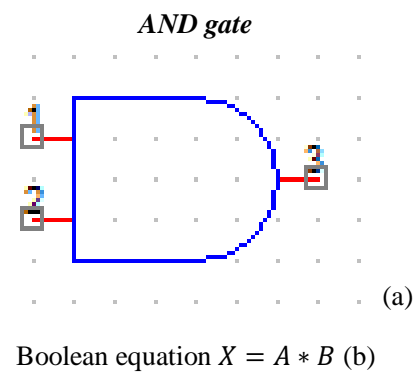

Truth table

\begin{tabular}{|c|c|}
\hline $\mathrm{A}$ & $\mathrm{B}$ \\
\hline 0 & 0 \\
\hline 0 & 1 \\
\hline 1 & 0 \\
\hline 1 & 1 \\
\hline
\end{tabular}

Figure 1. Example of AND logic gate symbol (a), Boolean equation (b) and truth table (c)

The custom written application uses QR code for identification. QR code is the trademark for a type of matrix barcode designed in 1994 for the automotive industry in Japan (Somayya \& Ramaswamy, 2015). A barcode is a machine-readable optical label that contains information about the item to which it is attached. The nature of QR code makes it ideal for a tracer for AR. In the AR the internal layout of the "NAND gates only" or "NOR only" for the corresponding logic gate is overlaid as on screen of the student.

A QR code is generated for each logic gate. It's so programmed that it would produce the truth table and Boolean equation if scanned with a smart device with any QR code scanning application. The AR of the "NAND gates only" or "NOR only" visualisations however will only function with the custom programmed application, provided to the students to download to their smart devices. The overlay of the AR shows the manufacturing layer of gates as based on the theory of the "NAND gates only" or "NOR gates only" manufacturing principle. Students are supposed to be able to take a design, convert and simplify it for a "sea of NAND gates" or "sea of NOR gates", which is an integral design step for integrated circuit layer manufacturing.

Below is an example of a laminated card with the QR code for the AND gate: 


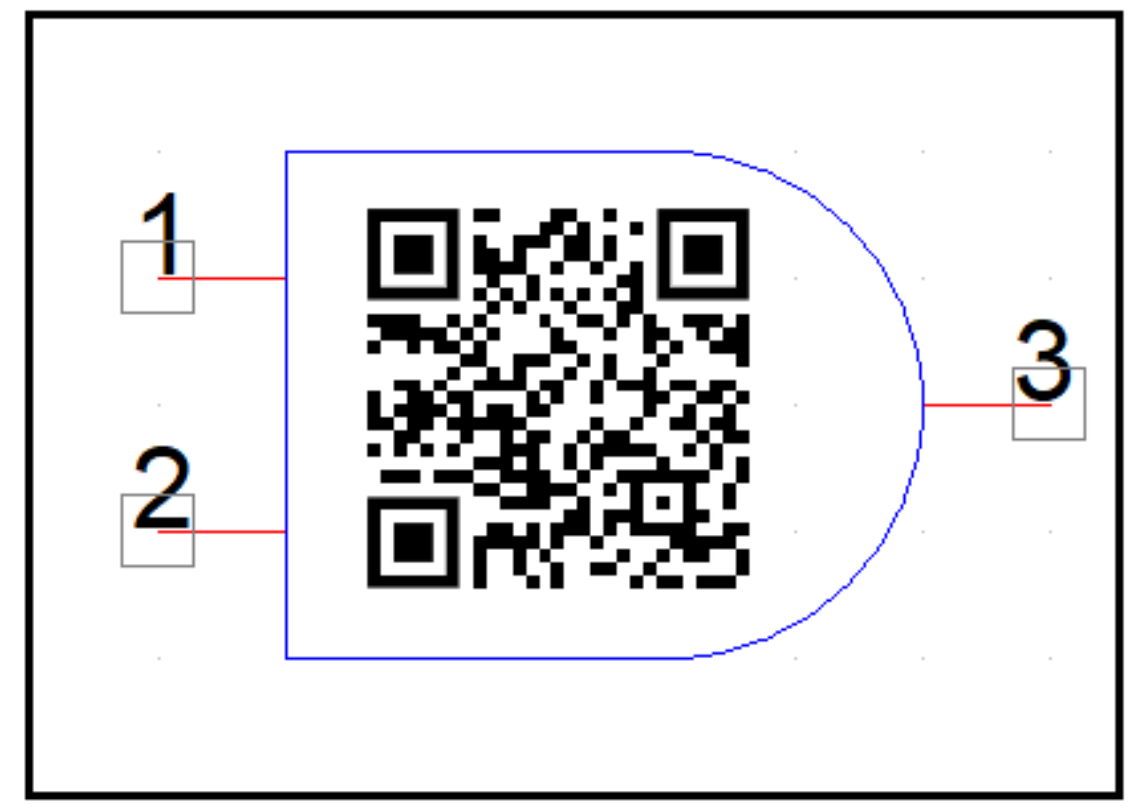

Figure 2. An example of a laminated card with the QR code for the AND gate

If scanned with any generic QR code scanner the truth table and equation is displayed.

The custom programmed AR app real-time example is as follows:

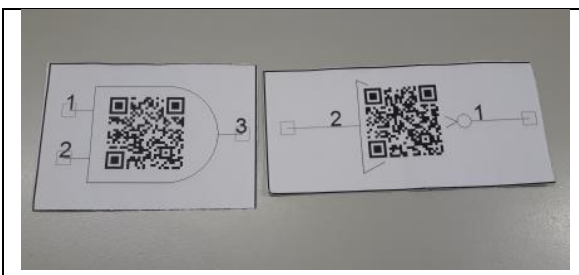

(a)

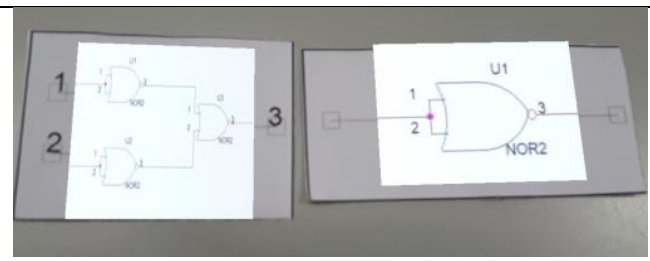

(b)

Figure 3. Example of the custom programmed AR application showing the cards before (a) and after (b) scan

This is an example for the "NOR gate only" substitution of gates. The APP runs on an android device. Note how it overlays the gates with their relevant substituted "NOR gates only" equivalents. In the case of XOR compiling where there needs to be some simplification steps, these simplification steps can be seen as an animation AR overlay. All overlays track the QR code and would move and orientate itself in real-time. 


\section{Methodology}

QR code and AR applications was programmed and installed on android devices and laminated cards made with logic gate symbols and codes as seen in figure 3 . This paper used an exploratory design which collected participants' perspectives of their experience during the QR code and AR activity, which is then analyzed with quantitative descriptions. Exploratory design means to understand a phenomenon better which has been previously not well understood (De Vos, Strydom, Fouche, \& Delport, 2011) while a descriptive analysis requires that the experience of the phenomenon is discovered (Foster, 2016). Data was collected with an online survey conducted directly after the augmented reality activity.

The target population of the study was the 52 students enrolled for the subject Logic Design III where the concept of logic gates was revisited and a sample of 18 completed the survey.

\section{Results}

Following is a breakdown of the results from the survey. Figure 4 and 5 was done before app and figure shows results after the app.

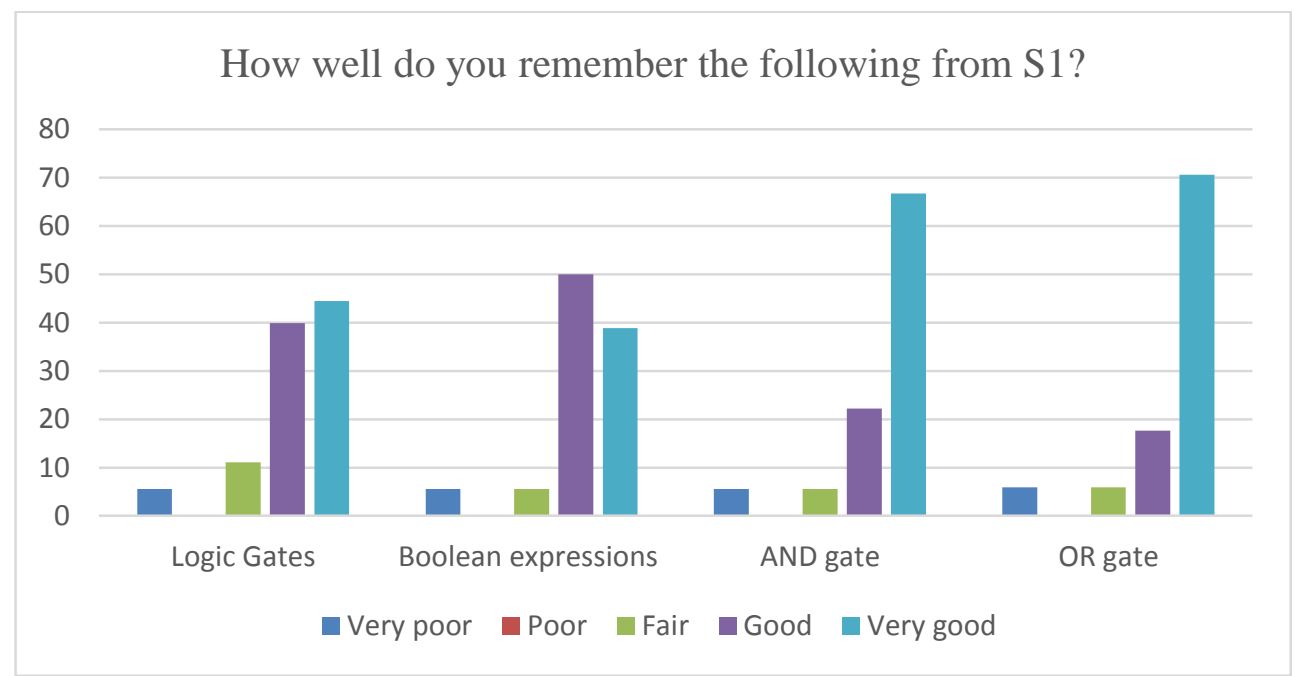

Figure 4. Participants' perspectives on how well they remember logic gates, Boolean equations, AND and OR gates.

Over all students indicated with a tendency towards good (38.89\%) and very good (44.44\%) that they remember the basic theory of digital logic gates. However, there is a very poor section as well (5.56\%) which is a matter of concern at this level. It is not overwhelmingly positive, and this is the "alphabet" of logic circuits especially for this level. 
How well do you remember the following from S1?

60

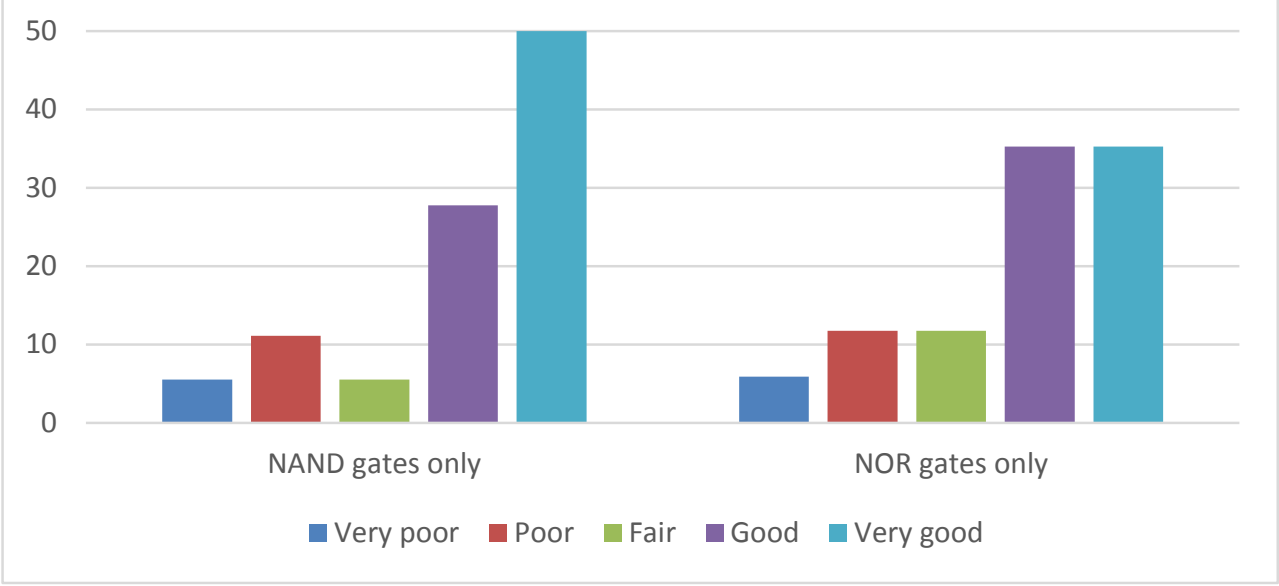

Figure 5. Participants' perspectives on how well they remember NAND and NOR gates only.

The "NAND gates only" and "NOR gates only" theory shows more uncertainty with $16.67 \%$ and $17.64 \%$ respectively admitting they do not remember that well which indicates the intervention should especially focus on these areas.

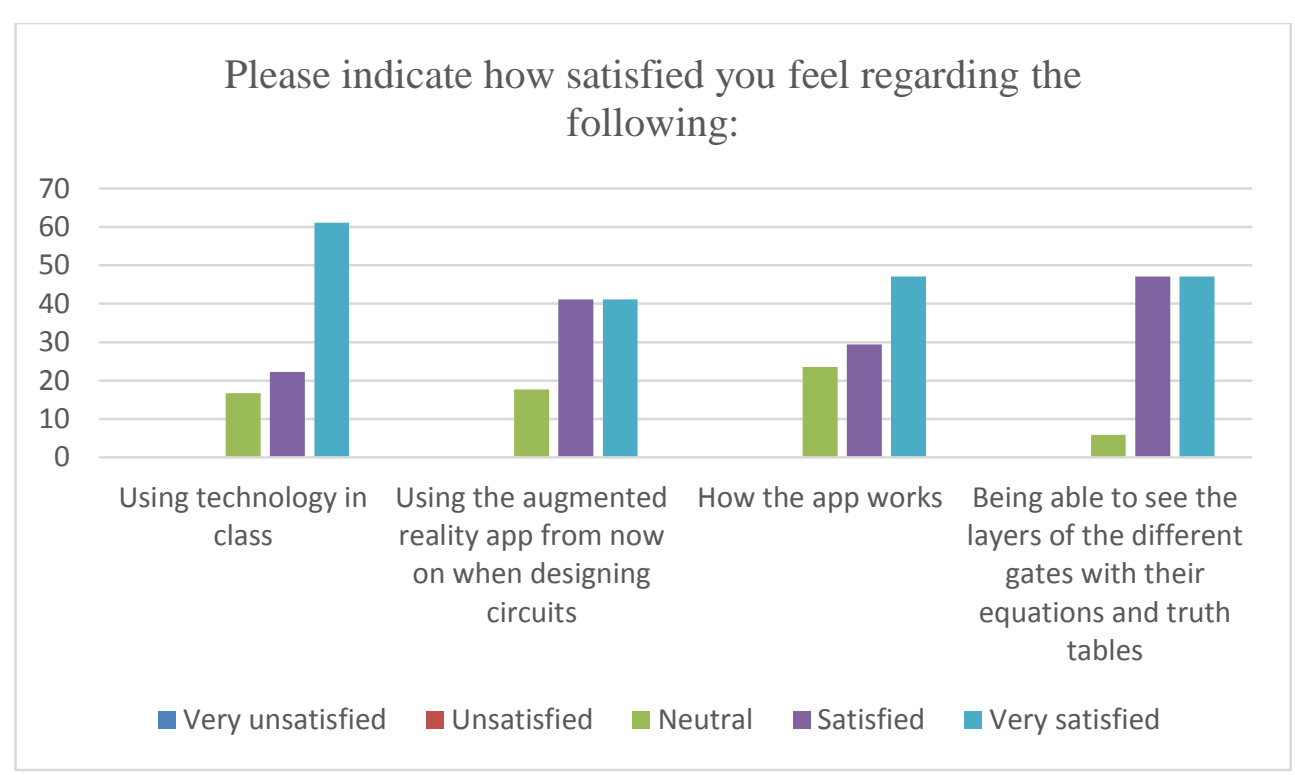

Figure 6. Participants' perspectives on the use of the AR application in class. 
Students indicated that they feel satisfied (22.22\%) to very satisfied $(61.11 \%)$ using technology in the class room indicating technology will be well received as a solution. The positive tendency also indicate that students would use the application and were very satisfied (41.18\% -using it and $47.06 \%$ - how it works) on the operation of it. They were mostly very satisfied $(47.06 \%)$ to be able to see equations, truth tables and layers with it.

Finally, when asked: "Do you think you will remember the different gates and their functions better if you use the app regularly?" A majority of $88.89 \%$ answered yes.

\section{Conclusion}

This paper investigated the perceptions of students on the use of AR to help them remember the concept of logic gates. The literature review showed that the facilitator is in part responsible for student success and that visual stimulus and technology could assist them to remember difficult concepts. Eighteen students completed a survey in this exploratory design to determine their feelings toward the technology and their memory of the concept from a previous semester. The $\mathrm{QR}$ and $\mathrm{AR}$ technologies were well received with one student saying: "This app would be useful because sometimes we are lazy to study but having to scan and get information you need would produce great results" and another pointing out that the use of technology suits the student of today by saying: "It's an interesting app to learn different gates. And in this generation, we prefer technology more than anything so it will be much easier to learn and memorize the work". The results show that most of the students felt they remembered logic gates from semester 1 still well enough for application in semester 4, which is contraindicative of what the facilitator experienced and may need deeper investigation. On the matter of the use of AR in the classroom the results showed very positive feelings $(83.33 \%)$ towards the adoption of this technology. The main result is $88.89 \%$ of students saying that they feel the technology will help them remember the difficult concept better with continuous use. Application of the software should be introduced at the earliest possible levels.

\section{References}

Alloghani, M., Al-Jumeily, D., Hussain, A., Aljaaf, A., Mustafina, J., \& Petrov, E. (2018). Healthcare Services Innovations Based on the State of the Art Technology Trend Industry 4.0. 2018 11th International Conference on Developments in eSystems Engineering (DeSE), (pp. 64-70).

Batcho, C. S., Gagne, M., Bouyer, L. J., Roy, J. S., \& Mercier, C. (2016). Impact of online visual feedback on motor acquisition and retention when learning to reach in a force field. Neuroscience, 337(1), 267-275. 
Cave, K. N. (2019, May 24). Dramatizing The Void: Crime Fiction's Journey to Forgetting. Retrieved from Andrews University https://digitalcommons.andrews.edu/cgi/viewcontent.cgi?article=1222\&context=arc

Chen, P., Liu, X., Cheng, W., \& Huang, R. (2016). A review of using Augmented Reality in Education. Innovations in Smart Learning, 13-18.

De Vos, A., Strydom, H., Fouche, C. B., \& Delport, C. S. (2011). Research at grass roots: For social sciences and human service professions. Pretoria: Van Schaik Publishers.

Floyd, T. L. (2015). Digital Fundamentals (11 ed.). New York: Pearson.

Foster, C. W. (2016). The lived persistent meaning of abrupt, permanent separation from a black, female nanny during early childhood: A phenomenological psychological study. Saybrook University.

Halpern, D. F., \& Hakel, M. D. (2003). Applying the science of learning to the university and beyond. Change, 35(4), 36-41.

Nazir, S., Totaro, R., Brambilla, S., Colombo, S., \& Manca, D. (2012). Virtual Reality and Augmented-Virtual Reality as Tools to Train Industrial Operators. Computer Aided Chemical Engineering, 1397-1401.

Somayya, M. R., \& Ramaswamy, S. T. (2015). Internet of Things (IoT): A Literature. Journal of Computer and Communications, 164-173. 